\title{
D $208 \quad$ Vortex Visualisation of an Actual Flight Soccer Ball
}

\author{
Takeshi ASAI (University of Tsukuba)
}

\begin{abstract}
The purpose of this study is to discuss the aerodynamic characteristics of soccer ball using a visualization method of the vortex structure around the real flight soccer ball in high Reynolds number. A visualisation experiment using titanium tetrachloride was conducted in order to visualise the flow around the soccer ball during flight. A soccer ball was placed directly in front of a soccer goal $25 \mathrm{~m}$ away and we had a subject perform a straight kick that involved virtually no rotation kick. A high speed video camera was set up at a midpoint between where the ball was placed and the soccer goal, and photographs were taken at 1,000 fps. By calculating the number of vortex shedding from this broad angle view, A horseshoe vortex on smooth sphere was observed in the range $380000<R e<1000000$ (Taneda, 1978). However, the vortex structure behind the soccer ball in super critical Re number region might be a distorted loop vortex.
\end{abstract}

Keywords: Soccer, Ball, Vortex, Re.

\section{Introduction}

Concerning the vortex structure of smooth sphere, Achenbach (1974) had shown that the complex vortex loops and sheets to a vortex configuration when the Reynolds number $(R e)$ is $10^{3}$, and when the Reynolds number is $5 \times 10^{4}$, the separation points will rotate. Taneda (1978) reported that when the Reynolds number is about $10^{4}$ to $3.8 \times 10^{5}$, the sphere wake performs a progressive wave motion in a plane containing the streamwise axis through the centre of the sphere. The plane rotates slowly and irregularly about that axis. The wave length equals about 4.5 times the sphere diameter. At Reynolds numbers ranging from $3.8 \times 10^{5}$ to $10^{6}$, the sphere wake forms a pair of streamwise line vortices at a short distance from the streamwise axis. The vortex pair rotate slowly and randomly about that axis. Sakamoto \& Haniu (1995) studied the vortex loop structure and its shedding process when the Reynolds number is $8.0 \times 10^{2}$ and above. Yun et al. (2003) had shown, using large eddy simulation for a Reynolds number of $10^{4}$, that the large-scale structure of the wake is in a spiral flow pattern. However, the vortex structure of a soccer ball is not clear on both the near-wake and the far-wake, and it is speculated that the vortex structure is highly related to the trajectory and dynamics.

The flight trajectory of a non-spinning or slowly spinning soccer ball may fluctuate in unpredictable ways. Such anomalous horizontal shaking or rapid falling is ascribed to a phenomenon called the 'knuckling effect' (Mehta, 1985). However, the aerodynamic properties and boundary-layer dynamics of the knuckling effect ball are not well-understood. The purpose of this study is to analyse the characteristics of vortex shedding and far-wake structure of the knuckling effect ball using high-speed video images and titanium tetrachloride (Asai et al., 2007).

\section{Methods}

A soccer ball was placed directly in front of a soccer goal $2 j$ $\mathrm{m}$ away and we had a subject perform a straight kick that involved virtually no rotation (Figure 1). That kicks were placement kicks delivered at the same velocity, as would occur in a real game. Two high-speed video cameras were set up at side of ball trajectory between where the ball was placed and the soccer goal, and photographs were taken at $1,000 \mathrm{fps}$ and a resolution of $1024 \times 1024$ pixels.

A visualisation technique using titanium tetrachloride was also conducted in order to visualise the flow around the soccer ball during flight. The experimental procedure was as follows. Each soccer ball was brush-painted with titanium tetrachloride, placed on a designated spot and then kicked towards a goal. As the ball flew towards the goal, the air flow around it was revealed by white smoke produced by the titanium tetrachloride. Photographs were taken using a high-speed video camera. Finally, the ball was collected and cleaned.

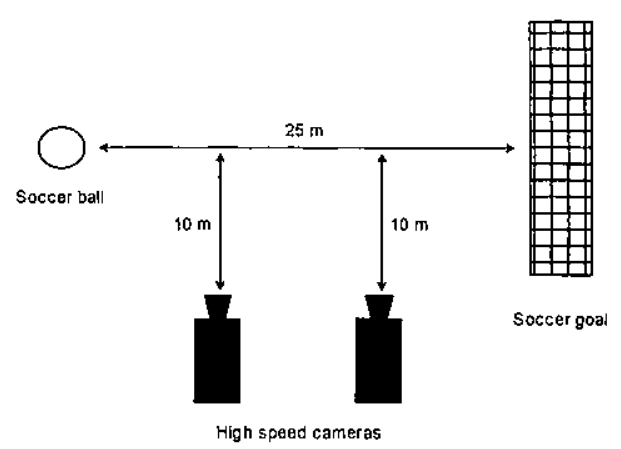

Fig. 1. Experiment set up 


\section{Results and Discussion}

Figure 2-a shows the large-scale vortex structure of a smooth sphere (diameter: $0.33 \mathrm{~m}$ ) set in a wind tunnel with Reynolds number of $2.4 \times 10^{4}$ (Taneda, 1978). The separation point of boundary layer is about 80 degrees from the front stagnation point of the sphere, and the wake shows a progressive wave motion, with a wavelength 4.5 times the diameter of the sphere. Taneda (1978) has reported that this progressive motion is not in a spiral pattern, but progresses along a flat surface on the sphere's symmetrical axis, and the vibrating surface is rotating irregularly and gently around the sphere's symmetrical axis. Also, in the case of a Reynolds number of $6.0 \times 10^{5}$, it is reported that the separation point of boundary layer largely recedes, and the dead air region shrinks (Figure 2-b). Taneda (1978) indicates that the wake forms two pieces of longitudinal vortex that rotate in opposite directions and are separated from the sphere's symmetrical axis. He also indicates that the vortex wake does not change its structure and rotates irregularly and gently around the sphere's symmetrical axis. On the other hand, in this experiment, the wake (far-wake) of a soccer ball that actually flies in low rotation has been observed with a distorted vortex trail, and in the large-scale structure, it shows the intermediate tendency of Figure 2-a and Figure 2-b (Figure 2-c). The Reynolds number for this experimental example was about $3.8 \times 10^{5}$ and is thought to be in the super-critical regime (Asai et al., 2007) beyond the critical Reynolds number $\left(\operatorname{Re}_{c r}=\sim 3.0 \times 10^{5}\right)$ of the soccer ball, but the above-mentioned large-scale structure was different from the vortex structure for the super-critical regime $\left(R e=6.0 \times 10^{5}\right)$ of a smooth sphere. This difference can be attributed to the difference in Reynolds number in the same super-critical regime, the difference in ball panel shapes, and the difference in surface roughness, but the details are to be further studied.

After balls that were under the influence of the knuckling effect were airborne, large-scale undulations of the vortex trail were observed. The frequency of these undulations ranged from $1: 0 \mathrm{~Hz}$ to $3.0 \mathrm{~Hz}$. Moreover, it is highly possible that the amount of movement that generates these undulations is related to the knuckling effect of the soccer ball. Further, we have noted that large-scale undulations of the vortex trail immediately suggest a possible dependence on the helical structure of the vortex in a ball under the influence of the knuckling effect.

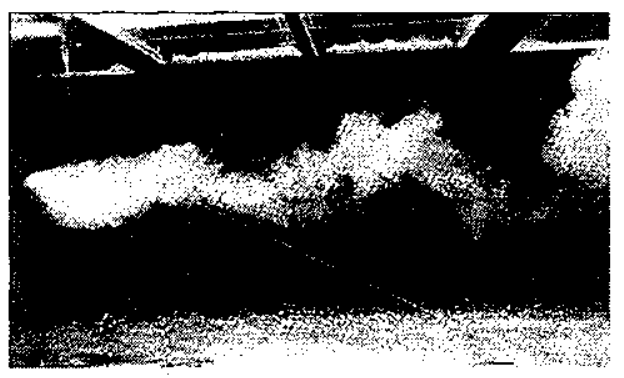

2-a (Taneda, 1978)

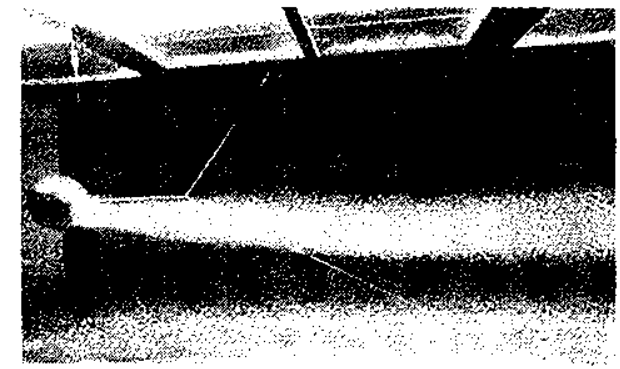

2-b (Taneda, 1978)

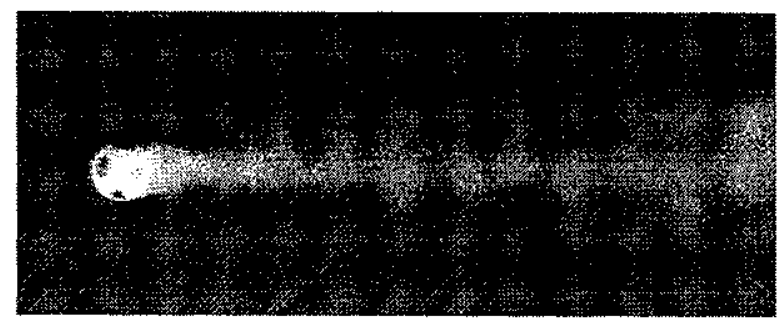

$2 \cdot \mathrm{c}$

Fig. 2. Vortex configuration of smooth sphere at $R e$ of $2.4 \times 10^{5}$ (a), that of smooth sphere at $R e$ of $6.0 \times 10^{5}$ (b) and that of soccer ball at $R e$ of $3.8 \times 10^{5}$ (c). Flow is from left to right.

\section{Conclusion}

The purpose of this study is to analyse the characteristics of vortex shedding and far-wake structure of the knuckling effect ball using high-speed video images and titanium tetrachloride. The wake (far-wake) of a soccer ball that actually flies in low rotation has been observed with a distorted vortex trail, and in the large-scale structure, it shows the intermediate tendency of the vortex configuration of smooth sphere at Reynolds numbers of $\sim 2.4 \times 10^{4}$ and that of $\sim 6.0 \times 10^{5}$.

\section{References}

Achenbach, E. (1974) Vortex shedding from sphere, Journal of Fluia Mechanics, 62, 209-221.

Asai, T., Seo, K., Kobayashi, O. \& Sakashita, R. (2007) Fundamental aerodynamics of the soccer ball, Sports Engineering, ISEA, 10(2), 101-109.

Mehta R. D. (1985) Aerodynamics of Sports Balls, Annual Review of Fluid Mechanics, 17, 151-189.

Sakamoto, H. \& Haniu, H (1995) The formation mechanics and shedding frequency of vortices from a sphere in uniform shear flow, Journal of Fluid Mechanics, 287, 151-171.

Taneda, S. (1978) Visual observations of the flow past a sphere at Reynolds numbers between $10^{\wedge} 4$ and $10^{\wedge} 6$. Journal of Fluid Mechanics, 85, 187-192.

Yun, G., Choi, H. \& Kim, D. (2003) Turbulent flow past a sphere at $\operatorname{Re}=3700$ and $10^{4}$, Physics of Fluids, 15-9, 6 . 